\title{
SOME STRENGTHENINGS OF THE ULAM NONMEASURABILITY CONDITION
}

\author{
S. MRóWKA
}

The purpose of this paper is to amplify the remarks made in the footnote on p. 603 in [4]. A complete account of the investigation initiated in [4] will be published in Fundamenta Mathematicae; in this paper we shall discuss various strengthenings of the Ulam nonmeasurability condition as well as their relative strength. Some of the present results were announced in [6].

We shall assume the familiarity with notations and terminology of [4] and [5]; we shall, however, review the more frequently used terms. By an Ulam measure in a set $X$ we shall mean a finitely additive $0-1$ measure $\mu$ whose domain is a field of subsets of $X$ containing all the one-element subsets of $X$ and such that $\mu(\{x\})=0$ for every $x \in X$ and $\mu(X)=1$. A measure on $X$ is a measure whose domain contains all subsets of $X$. Given a cardinal $\mathfrak{m}$ we will denote by $X_{\mathfrak{m}}$ a set of cardinality $\mathfrak{m}$ (in a topological context $X_{\mathfrak{m}}$ will denote a discrete space of cardinality $m)$. An infinite cardinal $m$ is said to be Ulam nonmeasurable provided that no Ulam measure on $X_{\mathfrak{m}}$ is countably additive. An Ulam measure $\mu$ is said to be $m$-additive provided that $\mu(\bigcup \Re)=\sup \{\mu(A): A \in \Re\}$ for every class $\Re$ of subsets of the domain of $\mu$ with card $\Re \leqq \mathfrak{m}$.

In [4] we have considered the following conditions on an infinite cardinal $\mathrm{m}$.

$\mathrm{m} \in M$ : there is a collection of sequences $A_{1}^{(\xi)}, A_{2}^{(\xi)}, \ldots ; \xi \in \Xi$, of subsets of $X_{\mathfrak{m}}$ such that card $\Xi \leqq \mathfrak{m}$ and for every Ulam measure $\mu$ on $X_{\mathfrak{m}}$ the equality

$$
\mu\left(\cup\left\{A_{n}^{(\xi)}: n=1,2, \cdots\right\}\right)=\sup \left\{\mu\left(A_{n}^{(\xi)}\right): n=1,2, \cdots\right\}
$$

fails for at least one $\xi \in \Xi$.

$S_{3}(\mathfrak{m}): X_{\mathfrak{m c l}} \subset \mathfrak{N}^{\mathfrak{m}}$.

$E H(\mathfrak{m}): \mathfrak{Y}^{\mathfrak{m}}$ is not $\mathfrak{m}$-compact.

( $\mathscr{T}$ denotes the space of nonnegative integers (i.e., a discrete space of cardinality $\left.\boldsymbol{N}_{0}\right) ; \mathfrak{r}^{\mathfrak{m}}$ is the topological product of $\mathfrak{m}$ copies of $\Re$. $m$-compact is used here in the following sense: if $\Omega$ is a class of closed subsets of a space such that card $\Omega=\mathfrak{m}$ and $\cap \Omega^{\prime} \neq \varnothing$ for every $\Omega^{\prime} \subset \Omega$ with card $\Omega^{\prime}<\mathfrak{m}$, then $\cap \Omega \neq \varnothing$.)

Each of the above conditions implies that $\mathfrak{m}$ is Ulam nonmeasura-

Received by the editors, August 15, 1968. 
ble. It was pointed out in [4] that these conditions are related as follows:

(a) $\mathfrak{m} \in M$ is equivalent to $S_{3}(\mathfrak{m})$;

(b) $S_{3}(\mathfrak{m})$ implies $E H(\mathfrak{m})$.

It was also pointed out in [4] that $E H(m)$ implies the following property of $\mathfrak{m}$ formulated in terms of logics with infinite expressions: there exists a system $\Sigma$ of $m$ first order sentences of countable length such that $\Sigma$ has no model but every subsystem $\Sigma^{\prime}$ of $\Sigma$ with card $\Sigma^{\prime}<\mathfrak{m}$ has one. This property was termed in [4] (for a lack of a better term) "very strong incompactness" of $\mathrm{m}$.

We do not know if implication (b) can be reversed.

In [4] we have also considered the following condition.

$S_{3}^{*}(\mathfrak{m}): X_{\mathfrak{m}}$ is homeomorphic to a closed subspace of a product of $\mathfrak{m}$ discrete spaces each of cardinality $<\mathfrak{m}$.

(This condition was denoted in [4] by $R(\mathfrak{m})$.) For an uncountable cardinal $\mathfrak{m}, S_{3}^{*}(\mathfrak{m})$ is obviously weaker than $S_{3}(\mathfrak{m})$. In a similar way we can weaken the conditions $\mathfrak{m} \in M$ and $E H(\mathfrak{m})$.

$\mathfrak{m} \in M^{*}$ : there is a collection of classes $\Re_{\xi} ; \xi \in \Xi$, of subsets of $X_{\mathfrak{m}}$ such that card $\Xi \leqq \mathfrak{m}$, card $\Re_{\xi}<\mathfrak{m}$ for every $\xi \in \Xi$, and for every finitely additive Ulam measure $\mu$ on $X \mathfrak{m}$ the equality $\mu\left(\cup \Re_{\xi}\right)=\sup \left\{\mu(A): A \in \Re_{\xi}\right\}$ fails for at least one $\xi \in \Xi$.

$E H^{*}(\mathfrak{m})$ : the product of $\mathfrak{m}$ discrete spaces, each of cardinality $<\mathfrak{m}$, need not be m-compact.

There is an essential difference between these two groups of conditions. Each of the conditions $\mathfrak{m} \in M, S_{3}(\mathfrak{m})$, and $E H(\mathfrak{m})$ is trivially true for $\mathfrak{m}=\boldsymbol{\aleph}_{0}$. In contrast, none of the conditions $\mathfrak{m} \in M^{*}, S_{3}^{*}(\mathfrak{m})$ and $E H^{*}(\mathfrak{m})$ is true for $\mathfrak{m}=\boldsymbol{\aleph}_{0}$. On the other hand, for an uncountable $\mathfrak{m}$, each of the conditions of the first group trivially implies the corresponding condition of the second group. Note, however, that the conditions of the second group do not imply that $m \in U$. In fact, these conditions are strengthenings of what can be called Ulam nonmeasurability in the extended sense. Following Banach [1], a measure that is $\mathfrak{n}$-additive for every $\mathfrak{n}<\mathfrak{m}$ will be called a measure of the type $\mathfrak{m}$. An uncountable cardinal $\mathfrak{m}$ is said to be Ulam nonmeasurable in the extended sense provided that there is no Ulam measure of the type $\mathfrak{m}$ on $X_{\mathfrak{m}}$; the class of all such cardinals will be denoted by $U^{*}$. It is easy to see that $U=U^{*} \cup\left\{\boldsymbol{N}_{0}\right\}$ iff $U$ contains all infinite cardinals iff $U^{*}$ contains all uncountable cardinals; if $U$ does not contain all infinite cardinals, then $U$ consists of all infinite cardinals that are smaller than the first uncountable one that is not in $U^{*}$. Each of the conditions $\mathfrak{m} \in M^{*}, S_{1}^{*}(\mathfrak{m})$, and $E H^{*}(\mathfrak{m})$ implies that $\mathfrak{m} \in U^{*}$.

Equivalence of the above conditions has been shown in the litera- 
ture for some cardinals. Results relevant for our discussion can be quoted as follows ( $\mathfrak{m}$ stands for an infinite cardinal and $\mathfrak{m}^{(\mathfrak{m})}$ stands for $\Sigma\left\{\mathfrak{m}^{\mathfrak{n}}: \mathfrak{n}<\mathfrak{m}\right\}$; definition of the class $C_{0}$ can be found on p. 709 of the present paper):

$\mathfrak{m} \in M^{*}$ and $\mathfrak{m} \in C_{0}$ are equivalent for an $\mathfrak{m}$ satisfying $\mathfrak{m}=\mathfrak{m}^{(\mathfrak{m})}$ [3, Theorem 4.31 (iii)].

$S_{3}^{*}(\mathfrak{m})$ and $\mathfrak{m} \in C_{0}$ are equivalent for an $\mathfrak{m}$ satisfying $\mathfrak{m}=\mathfrak{m}^{(\mathfrak{m})}$ [3, Theorem 4.32].

$E H^{*}(\mathfrak{m})$ implies $\mathfrak{m} \in \boldsymbol{C}_{0}$ for any $\mathfrak{m}[\mathbf{7}$, Lemma 1.7].

If $\mathfrak{m}$ is strongly inaccessible, then $\mathfrak{m} \in \boldsymbol{C}_{0}$ iff $\mathfrak{m}$ is strongly incompact [8, pp. 131-132].

We shall now show that the above conditions are equivalent for arbitrary cardinals.

TheOREM 1. For an uncountable cardinal $\mathfrak{m}$ the conditions $\mathfrak{m} \in M^{*}$, $S_{3}^{*}(\mathfrak{m}), E H^{*}(\mathfrak{m})$ are equivalent.

Proof. The proof of the equivalence of $m \in M^{*}$ and $S_{3}^{*}(\mathfrak{m})$ is the same as that of (a) indicated in [4]. In other words, the condition $\mathfrak{m} \in M^{*}$ is merely a set-theoretic translation of $S_{3}^{*}(\mathfrak{m})$ obtained via the general method of embedding into products given in [2]. Inasmuch as the conclusion of the method of [2] have now been formulated in a more detailed form in [5] it will be easier to refer to [5]. It is easy to see that we can assume that the classes $\Re_{\xi}$ occurring in the condition $\mathfrak{m} \in M^{*}$ are disjointed and that $U \Re_{\varepsilon}=X_{\mathfrak{m}}$. Such a class $\Re_{\xi}$ determines a (essentially unique) function $f_{\xi}$ of $X_{\mathfrak{m}}$ into a discrete space $X_{\xi}$ with card $X_{\xi}=$ card $\Re_{\xi}$ such that $\Re_{\xi}$ is the class of counterimages of points under $f_{\xi}$. By Theorem 2.1 in [5] the proof reduces to showing that the class $\left\{f_{\xi}: \xi \in \Xi\right\}$ is $\left\{X_{\xi}: \xi \in \Xi\right\}$-nonextendable iff the collection of the classes $\Re_{\xi}$ has the property expressed in the condition $\mathfrak{m} \in M^{*}$. This can be accomplished in the following way: let $c X_{\xi}$ be the one-point compactification of $X_{\xi}$; let $g_{\xi}$ be the continuous extension of $f_{\xi}$ with $g_{\xi}: \beta X_{\mathfrak{m}} \rightarrow c X_{\xi}$. Points of $\beta X_{\mathfrak{m}} \backslash X_{\mathfrak{m}}$ can be considered as Ulam measures on $X_{\mathfrak{m}}$; indeed, given a point $p \in \beta X_{\mathfrak{m}} \backslash X_{\mathfrak{m}}$ the formula $\mu_{p}(A)=0$ if $p \notin \bar{A}(\bar{A}$ denotes the closure of $A$ in $\beta X \mathfrak{m})$ and $\mu_{p}(A)=1$ if otherwise $\left(A \subset X_{\mathfrak{m}}\right)$ defines an Ulam measure $\mu_{p}$ on $X_{\mathfrak{m}}$; furthermore, $p \leftrightarrow \mu_{p}$ is a one-to-one correspondence between all the points of $\beta X_{\mathfrak{m}} \backslash X_{\mathfrak{m}}$ and all the Ulam measures on $X_{\mathfrak{m}}$. It is easy to see that for a point $p \in \beta X_{\mathfrak{m}} \backslash X_{\mathfrak{m}}$ we have $g_{\xi}(p) \notin X_{\xi}$ iff $\mu_{p}\left(\cup \Re_{\xi}\right) \neq$ $\sup \left\{\mu_{p}(A): A \in \Re_{\xi}\right\}$. The last statement combined with Theorem 2.1 in [5] completes the proof of the equivalence of $\mathfrak{m} \in M^{*}$ and $S_{3}^{*}(\mathfrak{m})$. In fact, we have shown a slightly stronger result: the cardinalities of the classes $\Re_{\xi}$ in the condition $m \in M^{*}$ are the same as the cardinalities of the discrete spaces whose product occurs in $S_{3}^{*}(\mathfrak{m})$. 
REMARK ON THE ABOVE PROOF. In view of an effective character of the parametric map occurring in Theorem 2.1 of [5] it is possible to translate [in a mechanical way] the above proof into purely settheoretic and product-theoretic terms. We shall give the end result of this translation-it is shorter but less natural. Assume that $\Re_{\xi}$ are the classes occurring in the condition $\mathfrak{m} \in M^{*}$; we shall assume that they are disjointed and that $U \Re_{\xi}=X_{\mathfrak{m}}$ for every $\xi \in \Xi$. Consider $\Re_{\xi}$ as discrete spaces; to every $x \in X_{\mathfrak{m}}$ we shall assign the point $r_{x}$ of the product $\otimes\left\{\Re_{\xi}: \xi \in \Xi\right\}$ defined by

$$
r_{x}(\xi)=\text { the only } A \text { with } x \in A \in \Re \xi .
$$

We shall show that

(i) for every $r_{0} \in \otimes\left\{\Re_{\xi}: \xi \in \Xi\right\}$ there are $\xi_{1}, \cdots, \xi_{n}$ such that $r_{0}\left(\xi_{1}\right) \cap \cdots \cap r_{0}\left(\xi_{n}\right)$ is finite.

Indeed, if (i) is false, then there exists a finitely additive Ulam measure $\mu$ on $X_{\mathfrak{m}}$ such that $\mu\left(r_{0}(\xi)\right)=1$ for every $\xi \in \Xi$ (extend the collection $\left\{r_{0}(\xi): \xi \in \Xi\right\}$ to a maximal filter not containing any finite set). Since $r_{0}(\xi) \in \Re_{\xi}$, we have, contrary to the assumption, $\sup \left\{\mu(A): A \in \Re_{\xi}\right\}=1=\mu\left(\cup \Re_{\xi}\right)$.

Now, the set $U=\left\{r: r\left(\xi_{i}\right)=r_{0}\left(\xi_{i}\right)\right.$ for $\left.i=1, \cdots, n\right\}$ is a neighborhood of $r_{0}$ and, by the definition of $r_{x}, r_{x} \in U$ implies $x \in r_{0}\left(\xi_{1}\right) \cap \ldots$ $\cap r_{0}\left(\xi_{n}\right)$. We have thus shown that every point $r_{0}$ of $\otimes\left\{\Re_{\xi}: \xi \in \Xi\right\}$ has a neighborhood $U$ such that $r_{x} \in U$ for only finitely many $x$. This implies that $1^{\circ}$ the set $\left\{r_{x}: x \in X_{\mathfrak{m}}\right\}$ is of cardinality $m$ (although we do not claim that $r_{x} \neq r_{x^{\prime}}$ for $\left.x \neq x^{\prime}\right)$ and $2^{\circ}$ this set is closed and discrete. Thus $\mathfrak{m} \in M^{*} \Rightarrow S_{3}^{*}(\mathfrak{m})$.

Conversely, assume that $X_{\mathfrak{m}}$ is a closed discrete subset of the product $\otimes\left\{X_{\xi}: \xi \in \Xi\right\}$, where card $\Xi=\mathfrak{m}$ and $X_{\xi}$ are discrete spaces each of cardinality $<\mathfrak{m}$. Define $A_{p}^{(\xi)}=\left\{x \in X_{\mathfrak{m}}: x(\xi)=p\right\}$ and $\Re_{\xi}=\left\{A_{p}^{(\xi)}\right.$ : $\left.p \in X_{\xi}\right\}$. Clearly, card $\Re_{\xi}<\mathfrak{m}$ and $\cup \Re_{\xi}=X_{\mathfrak{m}}$. Assume that $\Re_{\xi}$ 's fails to satisfy the property expressed in $\mathfrak{m} \in M^{*}$; i.e., there is a finitely additive measure $\mu$ on $X_{\mathfrak{m}}$ such that for every $\xi$ there is an $A \in R_{\xi}$ with $\mu(A)=1$. It is easy to check that the point $x_{0}$ of the product $\otimes\left\{X_{\xi}: \xi \in \Xi\right\}$ defined by $x_{0}(\xi)=$ the point $p$ of $X_{\xi}$ with $\mu\left(A_{p}^{(\xi)}\right)=1$, is an accumulation point of $X_{\mathfrak{m}}$. Thus $S_{3}^{*}(\mathfrak{m}) \Rightarrow \mathfrak{m} \in M^{*}$.

The implication $S_{3}^{*}(\mathfrak{m}) \Rightarrow E H^{*}(\mathfrak{m})$ is obvious; we shall prove that $E H^{*}(\mathfrak{m}) \Rightarrow S_{3}^{*}(\mathfrak{m})$. Let $X_{\xi}, \xi \in \Xi$, be discrete spaces each of cardinality $<\mathfrak{m}$, card $\Xi=\mathfrak{m}$; assume that the product $\otimes\left\{X_{\xi}: \xi \in \Xi\right\}$ is not $\mathfrak{m}$-compact. We can assume that $\Xi$ consists of all ordinals $\xi<\omega_{\lambda}$, where $\omega_{\lambda}$ is the initial ordinal of cardinality $\mathfrak{m}$; furthermore, we can assume (enlarging the spaces $X_{\xi}$ if necessary) that each $X_{\xi}$ contains all ordinals $\leqq \xi$. There is a collection of closed subsets $A_{\xi} ; \xi<\omega_{\lambda}$, of 
$\otimes\left\{X_{\xi}: \xi \in \Xi\right\}$ such that $\cap\left\{A_{\xi}: \xi<\omega_{\lambda}\right\}=\varnothing$ and $\cap\left\{A_{\eta}: \eta<\xi\right\} \neq \varnothing$ for every $\xi<\omega_{\lambda}$. It follows that for every $\xi<\omega_{\lambda}$ we can find a point $x_{\xi} \in$ $\otimes\left\{X_{\xi}: \xi \in \Xi\right\}$ such that $x_{\xi}(\eta)=y_{\xi}(\eta)$ for every $\eta<\xi$, where $y_{\xi}$ is some point of $\bigcap\left\{A_{\eta}: \eta<\xi\right\}$, and $x_{\xi}(\eta)=\xi$ for $\eta \geqq \xi$. It is easy to verify that $\left\{x_{\xi}: \xi \in \Xi\right\}$ is a closed discrete subspace of $\otimes\left\{X_{\xi}: \xi \in \Xi\right\}$ of cardinality $\mathfrak{m}$.

Theorem 1 is shown.

We shall now prove that $E H^{*}(\mathfrak{m})$ implies that $\mathfrak{m}$ is "strongly incompact". This proof is identical with that showing that $E H(\mathfrak{m})$ implies that $m$ is "very strongly incompact".

Let $X_{\xi}$ and $A_{\xi}, \xi \in \Xi$, have the same properties as in the proof of Theorem 1. We shall first translate these properties into set-theoretic terms. $A_{\xi}$ is a collection of functions defined on $\Xi$ with the property

(ii) if $x \in \otimes\left\{X_{\xi}: \xi \in \Xi\right\}$ and for every finite subset $\Xi_{0}$ of $\Xi$ there is a $y \in A_{\xi}$ with $x\left|\Xi_{0}=y\right| \Xi_{0}$, then $x \in A_{\xi}$.

For every finite subset $\Xi_{0}$ of $\Xi$ we denote by $A_{\xi, \Xi_{0}}$ the collection of all the restrictions $X \mid \Xi_{0}$, where $x \in A$. Note that card $A_{\xi, \Xi_{0}}<\mathrm{m}$. Consider now the logic $L_{\mathfrak{m}}$ (with the equality) having $\cup\left\{X_{\xi}: \xi \in \Xi\right\} \cup \Xi$ as the set of constants (we assume here that $U\left\{X_{\xi}: \xi \in \Xi\right\} \cap \Xi=\varnothing$ ) and having one function symbol $f$. For every $\xi \in \Xi$ we shall take a sentence $\tau_{\xi}$ asserting that the value of $f$ at $\xi$ is an element of $X_{\xi} ; \tau_{\xi}$ is a disjunction of fewer than $m$ atomic sentences (equalities). Furthermore, for every $\xi \in \Xi$ and every finite subset $\Xi_{0}$ of $\Xi$ we shall take a sentence $\sigma_{\xi, \Xi_{0}}$ asserting that the restriction of $f$ to $\Xi_{0}$ belongs to $A_{\xi, \Xi_{0}}$. Since card $A_{\xi, \Xi_{0}}<\mathrm{m}, \sigma_{\xi, \Xi_{0}}$ is a disjunction of fewer than $\mathrm{m}$ finite conjunctions. It takes only calligraphy to write down these sentences explicitly. The system $\Sigma$ of all the $\tau_{\xi}$ 's and all the $\sigma_{\xi, \Xi_{0}}$ 's has the desired properties (strictly speaking, one should adjoin to $\Sigma$ some trivial sentences, like those asserting that all the involved constants are distinct, etc.). Note the formal simplicity of $\Sigma$. It consists of open sentences and, besides the constants, it involves only one nonlogical symbol. Of course, a function symbol could be replaced by a symbol of a binary relation; in this case some other trivial sentences would have to be adjoined to $\Sigma$.

Concluding Remarks. 1. Conditions $\mathfrak{m} \in \boldsymbol{M}$ and $\mathfrak{m} \in \boldsymbol{M}^{*}$ are formulated in terms of finitely additive Ulam measures. It was pointed out in the footnote on p. 603 in [4] that various strengthenings of the Ulam nonmeasurability condition can be formulated in terms of countably additive measures. We shall briefly discuss the approach, although it does not seem that it can lead to mathematically interesting results. Roughly speaking, these conditions refer to the following: how many subsets of $X_{\mathfrak{m}}$ one has to select so that given any countably additive Ulam measure $\mu$ in $X_{\mathfrak{m}}$ at least one of the selected sets is 
$\mu$-nonmeasurable (i.e., is not in the domain of $\mu$ )? Precisely, consider a countably additive Ulam measure $\mu$ in $X_{\mathfrak{m}}$ such that the domain of $\mu$ is $\mathfrak{m}^{\prime}$-additive for every $\mathfrak{m}^{\prime}<\mathfrak{m}$. Let $T(\mathfrak{m}, \mathfrak{n})$ stand for: there is a class $\Re$ of subsets of $X_{\mathfrak{m}}$ such that card $\Re \leqq \mathfrak{n}$ and for every $\mu$ as above at least one of the sets in $\Omega$ is not $\mu$-measurable. $T^{*}(\mathfrak{m}, \mathfrak{n})$ is formulated in the same way except that now $\mu$ is assumed to be of the type $\mathfrak{m}$. $T(\mathfrak{m}, \mathfrak{n})$ and $T^{*}(\mathfrak{m}, \mathfrak{n})$ are monotone relative to the second term; i.e., for $\mathfrak{n}^{\prime}>\mathfrak{n}, T(\mathfrak{m}, \mathfrak{n})$ is stronger than $T\left(\mathfrak{m}, \mathfrak{n}^{\prime}\right)$ and $T^{*}(\mathfrak{m}, \mathfrak{n})$ is stronger than $T^{*}\left(\mathfrak{m}, \mathfrak{n}^{\prime}\right)$. A cardinal $\mathfrak{m}$ is Ulam nonmeasurable iff $T\left(\mathfrak{m}, 2^{\mathfrak{m}}\right)$ holds, (similarly, $\mathfrak{m} \in U^{*}$ iff $T^{*}\left(\mathfrak{m}, 2^{\mathfrak{m}}\right.$ ) holds). Consequently, we can formulate various strengthenings of the Ulam nonmeasurability conditions with the aid of $T(\mathfrak{m}, \mathfrak{n})$; for instance, the condition " $T(\mathfrak{m}, \mathfrak{m})$ holds" is one of them. In fact, most of the cardinals in $U$ satisfy $T(\mathfrak{m}, \mathfrak{n})$. However, $T(\mathfrak{m}, \mathfrak{m})$ does not express the strongest property of cardinals; indeed, an analysis of the Ulam proof in [7] shows that $T\left(2^{\mathfrak{n}}, \mathfrak{n}\right)$ holds for every $\mathfrak{n} \in U$ (and $T^{*}\left(2^{\mathfrak{n}}, \mathfrak{n}\right)$ holds for every infinite $\mathfrak{n})$. It does not seem that this procedure can lead to conditions that are significantly stronger than the original nonmeasurability condition. Note that some conditions formulated in this way have been studied in the literature; for instance, the condition $\mathfrak{m} \in C_{0}$ of [3] is equivalent to $T^{*}(\mathfrak{m}, \mathfrak{m})$.

2. The role of the axiom of choice in the above considerations can be of interest. The following are sample questions related to the condition $S_{3}(\mathfrak{m})$. If the axiom of choice is not assumed one has to be careful how $\mathfrak{T}^{\mathfrak{m}}$ is defined. We shall assume one of the standard axiomatics of set-theory with the full axiom of extensionality; ordinals are meant in the von Neumann sense; $\mathscr{N}$ is the set of all finite ordinals and $\mathfrak{x}^{\mathfrak{m}}$ is the set of all functions into $\mathscr{r}$ defined on a set of cardinality $\mathfrak{m}$ (the concept of a cardinal number remains, in fact, undefined; in these considerations we use only the phrase: "two sets are of the same cardinality"). We do not know if $S_{3}\left(\boldsymbol{\aleph}_{1}\right)$ can be proved without the axiom of choice. More precisely, we do not know if $X_{\aleph_{1} \text { cl }} \cup N^{N_{1}}$ can be proved without the axiom of choice. On the other hand, $X_{\aleph_{1} \text { l }} \subset \otimes\left\{S(\xi): \omega_{0} \leqq \xi<\omega_{1}\right\}$ can be proved without the axiom of choice $(S(\xi)$ denotes here the discrete space consisting of all ordinals $<\xi)$. Of course, the axiom of choice implies that $\mathfrak{r}^{\aleph_{1}}$ and $\otimes\left\{S(\xi): \omega_{0} \leqq \xi<\omega_{1}\right\}$ are homeomorphic; but we do not know if the axiom of choice can be eliminated here (note that the axiom of choice is not needed to prove that $\Re{ }^{N_{1}}$ and $\otimes\left\{S(\xi): \omega_{0} \leqq \xi<\omega_{1}\right\}$ are of the same cardinality). Similar comments apply to other cardinals of the form $\boldsymbol{N}_{\alpha+1}$.

As far as cardinals of the form $2^{\mathrm{m}}$ are concerned we shall prove the following. 
THEOREM 2. If $\mathfrak{m}$ is an aleph, then $X_{2 \mathrm{~m}_{\mathrm{c} 1}} \subset X^{2^{\mathfrak{m}}}$ can be proved effectively.

Proof. The proof is a translation of the Ulam procedure [9] obtained via the methods of [2] and [4]; we shall only give the end result of this translation (compare with the remark in the proof of Theorem 1). Assume that $X_{\mathfrak{m}}$ consists of all ordinals $<\omega_{\lambda}$; let $\Xi_{0}$ be the set of all functions on $X_{\mathfrak{m}} \backslash\{0\}$ with values 0 and 1 ; let $\Xi=\Xi_{0}$ $\mathrm{U}\left(X_{\mathfrak{m}} \backslash\{0\}\right)$. $\Xi$ is effectively of cardinality $2^{\mathfrak{m}} . X_{\mathfrak{m}}^{2^{\mathfrak{m}}}$ will be treated as the set of all functions from $\Xi$ in to $X_{\mathfrak{m}}$; elements of $X_{\mathfrak{m}}^{2 \mathfrak{m}}$ can be written as pairs $(a, \xi)$, where $a$ and $\xi$ are functions on $\Xi_{0}$ and $X_{\mathfrak{m}} \backslash\{0\}$, respectively. To every $\xi \in \Xi_{0}$ we shall attach the element $x_{\xi}=\left(a_{\xi}, \xi\right)$ of $X_{\mathfrak{m}}^{2 \mathfrak{m}}$, where $a_{\xi}$ is defined as follows:

$$
a_{\xi}(\xi)=0 \text {, if } \xi \neq \sigma \text {, then } a_{\xi}(\sigma)=\text { the first } \gamma \text { in } X_{\mathfrak{m}} /\{0\} \text { with } \xi(\lambda) \neq \sigma(\lambda) \text {. }
$$

The set $\tilde{X}=\left\{x_{\xi}: \xi \in \Xi_{0}\right\}$ is of cardinality $2^{\mathrm{m}}$. We shall prove that every point $x^{(0)}$ of $X^{{ }^{\mathfrak{m}}}$ has a neighborhood which contains at most one point of $\tilde{X}$. Let $x^{(0)}=\left(a^{(0)}, \xi^{(0)}\right)$. If at least one coordinate of $\xi^{(0)}$ is $>1$, then there is nothing to prove. We can therefore assume that $\xi^{(0)} \in \Xi_{0}$. We shall distinguish two cases.

1st case. $a^{(0)}\left(\xi^{(0)}\right)=0$. We let $U=\left\{(a, \xi): a\left(\xi^{(0)}\right)=0\right\}$. Note that if $\xi \neq \xi^{(0)}$, then $a_{\xi}\left(\xi^{(0)}\right) \neq 0$. Therefore $U \cap \tilde{X}=\left\{x_{\xi}(0)\right\}$.

2nd case. $a^{(0)}\left(\xi^{(0)}\right)=\lambda_{0} \neq 0$. We let

$$
\begin{aligned}
U=\left\{(a, \xi): a\left(\xi^{(0)}\right)=\lambda_{0}\right\} \backslash\{(a, \xi): \xi(\lambda) & =\xi^{(0)}(\lambda) \\
& \text { for } \left.0<\lambda<\lambda_{0} \text { and } \xi\left(\lambda_{0}\right) \neq \xi^{(0)}\left(\lambda_{0}\right)\right\} .
\end{aligned}
$$

The second term of the above difference is closed; consequently $U$ is a neighborhood of $x^{(0)}$. Assume that $x_{\xi}=\left(a_{\xi}, \xi\right) \in U$. Then $a_{\xi}\left(\xi^{(0)}\right)=\lambda_{0}$, hence $\lambda_{0}$ is the first element for which $\xi\left(\lambda_{0}\right) \neq \xi^{(0)}\left(\lambda_{0}\right)$; therefore $\xi(\lambda)$ $=\xi^{(0)}(\lambda)$ for $0<\lambda<\lambda_{0}$; but this implies that $\left(a_{\xi}, \xi\right) \notin U$. Thus $U \cap \tilde{X}$ $=\varnothing$.

(Note that we have defined [effectively] a function $\phi$ on $\tilde{X}$ such that, for every $x \in \tilde{X}, \phi(x)$ is a finite subset of $\Xi$, and every $x^{\prime} \in \tilde{X}$ with $x \neq x^{\prime}$ differs from $x$ on some element of $\phi(x)$. The set $\tilde{X}$ could be therefore called effectively discrete. However, it is impossible to produce a subset of $X_{\mathfrak{m}}^{2 \mathfrak{m}}$ which is both effectively discrete and effectively closed ["effectively closed" being defined in a similar way] unless $2^{\mathrm{m}}$ is an aleph.)

It follows from Theorem 2 that $X_{2 \mathrm{~N}_{0} \mathrm{cl}} \subset N^{2 \mathrm{~N}_{0}}$ can be shown effectively. Similarly, to prove effectively that $X_{2^{c}}{ }^{c l} \subset N^{2^{c}}$ it suffices to assume that $c=2^{N_{0}}$ is an aleph. 


\section{BIBLIOGRAPHY}

1. S. Banach, Über additive Massfunktionen in abstraken Mengen, Fund. Math. 15 (1929), 97-101.

2. R. Engelking and S. Mrowka, On E-compact spaces, Bull. Acad. Polon. Sci. Sér. Sci. Math. Astronom. Phys. 6 (1958), 429-436. MR 20 \#3522.

3. H. J. Keisler and A. Tarski, From accessible to inaccessible cardinals. Results holding for all accessible cardinal numbers and the problem of their extension to inaccessible ones, Fund. Math. 53 (1963/64), 225-308. MR 29 \#3385.

4. S. Mrówka, On E-compact spaces. II, Bull. Acad. Polon. Sci. Ser. Sci. Math. Astronom. Phys. 14 (1966), 597-605. MR 34 \#6712.

5. - Further results on E-compact spaces. I, Acta. Math. 120 (1968), 161185. MR 37 \#2165.

6. - Some strengthenings of the Ulam non-measurability condition, Notices Amer. Math. Soc. 14 (1967), 247. Abstract \#643-20.

7. D. Monk and D. Scott, Additions to some results of Erdös and Tarski, Fund Math. 53 (1963/64), 335-343. Mr 29 \#3386.

8. A. Tarski, Some problems and results relevant to the foundations of set theory. Logic, methodology and philosophy of science, Proc. Internat. Congress (Stanford University, Stanford, Calif., 1960) Stanford Univ. Press, Stanford, Calif., 1962, pp. 125135. MR $27 \# 1382$.

9. S. Ulam, Zur Masstheorie in der allgemeinen Mengenlehre, Fund. Math. 16 (1930), 140-150.

The Pennsylvania State University, University Park, Pennsylvania 16802 